\title{
Protective effect of dapagliflozin on colistin-induced renal toxicity
}

\author{
Ejder Saylav Bora ${ }^{1, \star}$, Mumin Alper Erdogan ${ }^{2}$, Ayfer Meral ${ }^{3}$, Zeynep Karakaya ${ }^{1}$, \\ Oytun Erbas ${ }^{4}$
}

\author{
${ }^{1}$ İzmir Katip Çelebi University Atatürk \\ Research and Training Hospital, \\ Emergency Medicine, İzmir, Turkey \\ ${ }^{2}$ İzmir Katip Çelebi University, Faculty of \\ Medicine, Department of Physiology, \\ Izmir, Turkey \\ ${ }^{3}$ Van Yüzüncü Yıl University Department \\ of Biochemistry Van, Turkey \\ ${ }^{4}$ Demiroğlu Bilim University Depatment \\ of Physiology Istanbul, Turkey
}

\section{*Correspondence}

saylavbora@hotmail.com

(Ejder Saylav Bora)

\begin{abstract}
Objectives: Multiple-drug resistance to Gram-negative bacteria has increased significantly in recent years. Colistin is increasingly used as a last line of defense against these bacteria. However, colistin has been associated with nephrotoxicity in experimental animals. This study explores the protective effect of dapagliflozin in a rodent model of nephrotoxicity.

Material Method: The present study includes a total of 24 male rats, of which 16 were given a single $20 \mathrm{mg} / \mathrm{kg}$ dose of colistin (Colimycin $150 \mathrm{mg} / \mathrm{mL}$ ) intravenously to induce renal toxicity. The remaining eight rats were given no drugs in order to serve as the control, Group A. The 16 rats treated with colistin were then divided into two groups. Rats in Group B received $0.9 \% \mathrm{NaCl}$ saline solution at a dose of $30 \mathrm{~mL} / \mathrm{kg} / \mathrm{day}$ intraperitoneally (i.p.) and $10 \mathrm{mg} / \mathrm{kg} /$ day dapagliflozin (Forziga $10 \mathrm{mg}$ ) via oral gavage. Those in Group C received $0.9 \% \mathrm{NaCl}$ saline solution at an i.p. dose of $30 \mathrm{~mL} / \mathrm{kg} / \mathrm{day}$. Both saline and dapagliflozin were administered as described over the course of ten days. The animals were euthanized and blood samples were taken by cardiac puncture for further analysis. Their kidneys were removed for histopathological and biochemical examination.

Results: Levels of creatinine, BUN, KIM-1, and MDA were significantly increased in the 16-rat (Groups B and C) treatment group, in comparison to the control group; however, these biomarkers were significantly normalized in Group B, which had received dapagliflozin in addition to saline. The GSH levels of Group C showed significant decline when compared to those of the control group, and were significantly normalized in Group B. Histologically, in Group 2, we observed severe tubular dilatation and tubular epithelial cell injury in comparison to the control group. These severe anatomical changes were decreased in Group B.

Conclusion: Apart from its positive effect on glucose regulation, which is the usual purpose of dapagliflozin, we observed that in colistin-induced nephrotoxicity, it decreases oxidative stress by inhibiting SGLT-2, and has restorative effects in terms of histopathology and biochemistry. These findings offer hope that the use of dapagliflozin may be protective for contrast nephropathy, which causes renal tubule damage through oxidative mechanisms. Future studies will further clarify the mechanistic action of colistin and dapagliflozin, and may support the hypothesis that dapagliflozin can be used as an adjunctive therapy in all nephrotoxic conditions.
\end{abstract}

\section{Keywords}

Nephrotoxicity; Colistin; Dapagliflozin; Histopathology

\section{Introduction}

Colistin is a cyclic-structured antibiotic that has rarely been used until recent years, due to its dose-dependent nephrotoxicity side effects [1]. Multiple antibiotic resistance has begun to occur in patients admitted to intensive care, and in this context, the usage of colistin has increased, playing an important role in patients' clinical recoveries and survival.
Colistin affects the proximal tubule and increases the permeability of the membrane, resulting in increased passage of water and electrolytes. This causes an increase in the blood creatinine parameter, glucosuria, proteinuria, and hematuria; acute tubular necrosis consequently occurs by cytotoxic effect [2]. Nephrotoxicity due to colistin [3] is reversible. Generally, kidney laboratory values return to normal levels within one month [4]. Restrictions in the application of dose-dependent 
nephrotoxic colistin have revealed the need for a nephroprotective drug for use in conjunction with colistin. The nephrotoxic mechanism of colistin has yet to be elucidated. This situation has increased the number of studies on the availability of drugs to reduce or halt colistin nephrotoxicity.

The SGLT-2 inhibitor dapagliflozin is used for treating diabetes mellitus (DM). It received FDA approval in January, 2014 [5]. The inhibition of SGLT2 serves to regulate glucose concentration independently from insulin by augmenting renal glucose excretion. Plasma glucose concentration increases comparably with renal glucose filtration. Thus, by increasing glucosuria with dapagliflozin, insulin deficiency and thus hyperglycemia are compensated for [5].

Reactive oxygen species released due to oxidative stress are thought to be the main cause of drug-dependent renal tubular damage. One study shows that hyperfiltration, tubular oxidative stress, and oxygen consumption in a diabetic kidney are diminished by SGLT2 inhibition, which alleviates kidney damage [6]. In this study, we investigated the corrective effect of dapagliflozin on the impaired values of colistininduced nephrotoxicity, evaluating its appropriateness as a nephroprotective agent.

\section{Materials and methods}

\subsection{Animal care and experiments}

Twenty-four male Sprague Dawley rats each weighing between 200 and $220 \mathrm{~g}$ were selected. Rats were nourished ad libitum and housed in sets in steel cages having a temperaturecontrolled environment $\left(22 \pm 2{ }^{\circ} \mathrm{C}\right)$ with 12 -h light/dark cycles. All experimental studies described here were approved by the Animal Ethics Committee, and were conducted according to the Guidelines for the Care and Use of Laboratory Animals, as approved by the National Institutes of Health (USA).

\subsection{Experimental protocol}

Of the 24 male rats were included in the study, 16 were given a single $20 \mathrm{mg} / \mathrm{kg}$ dose of colistin (Colimycin $150 \mathrm{mg} / \mathrm{mL}$ ) to induce renal toxicity. The remaining eight rats were given no drugs and comprise the control (Group A). Next, the 16 rats treated with colistin were separated into two groups:

Group B: Rats given $0.9 \% \mathrm{NaCl}$ isotonic intraperitoneally (i.p.) at a $30 \mathrm{~mL} / \mathrm{kg} /$ day dose, and a $10 \mathrm{mg} / \mathrm{kg} /$ day dose of dapagliflozin (Forziga $10 \mathrm{mg}$ ) via oral gavage.

Group C: Rats given only $0.9 \% \mathrm{NaCl}$ isotonic given i.p. at a dose of $30 \mathrm{~mL} / \mathrm{kg} /$ day.

Both Group B and Group C received saline and dapagliflozin for 10 days. The animals were then euthanized and blood samples were obtained by cardiac puncture for further analysis. Their kidneys were also removed for histopathological and biochemical assessment.

\subsection{Histopathological examination of kidney}

In the histological and immunohistochemical portion of the study, ketamine $\left(80 \mathrm{mg} / \mathrm{kg}\right.$, Alfamine ${ }^{\circledR}$, Alfasan International B.V., Netherlands) and xylazine $\left(10 \mathrm{mg} / \mathrm{kg}\right.$, Alfazyne ${ }^{\circledR}, \mathrm{Al}-$ fasan International B.V., Netherlands) i.p. sedoanalgesia was perfused with $200 \mathrm{~mL}$ of $4 \%$ formaldehyde in $0.1 \mathrm{M}$ phosphate buffered saline (PBS). Kidney sections $(4 \mu \mathrm{m})$ were obtained after fixing with formalin and stained with hematoxylin and eosin. All sections were photographed with the Olympus BX51 microscope and Olympus C-5050 digital camera.

The morphological study was performed with a computerized image analysis system (Image Pro Express 1.4.5, Media Cybernetics, Inc. USA), by an observer blinded to the study group, on 10 microscopic fields per section, at $20 \times$ magnification. Tubular injury score was previously described [7]. In summary, tubular damage was determined through observations of tubular vacuolation and tubular epithelial necrosis, and was scored by grading the percentage of affected tubules counted under 400 magnification. Injured tubules score were considered as standard under 400 magnification expressed in tubular numbers per field. Ranking grading in each area was calculated as follows:

Injury score $(\%)=$ (numbers of injured tubules/number of whole tubules) 100 .

A number of 10 or more areas in the cortex per slide were randomly selected.

\subsection{Measurement of kidney injury molecule-1 (KIM-1), BUN and creatinine levels}

For all animals, blood levels of urea and creatinine were measured using the Olympus AO5800 auto analyzer with enzymatic method. The biomarker for human kidney damage, Kidney Injury Molecule-1 (KIM-1), augmented by injury in the proximal tubule, was measured using ELISA.

\subsection{Measurement of tissue lipid peroxidation (MDA)}

Lipid peroxidation was determined in tissue samples by measuring malondialdehyde (MDA) levels, which is defined as the marker of oxidative stress, and can be measured using a thiobarbituric acid reactive substances (TBARS) assay [8]. Briefly, trichloroacetic acid and TBARS reagent were added to the tissue samples, then mixed and incubated at $100{ }^{\circ} \mathrm{C}$ for $60 \mathrm{~min}$. After cooling on ice, the samples were centrifuged at $3000 \mathrm{rpm}$ for 20 minutes, and the absorbance of the supernatant was read at $535 \mathrm{~nm}$. MDA levels were calculated from the standard calibration curve using tetraethoxypropane and expressed as $\mathrm{nmol} / \mathrm{gr}$ protein.

\subsection{Measurement of tissue protein levels}

Total protein concentration in kidney samples was determined by Bradford's method using bovine serum albumin as usual [9].

\subsection{Determination of tissue glutathione (GSH) levels}

The content in tissue samples of GSH, the indicator of cellular defense component of cells, was measured spectrophotometrically according to Ellman's method [10]. In this method, thiols interact with 5, 5'-dithiobis- (2-nitrobenzoic acid) (DTNB) and form a colored anion with maximum peak at $412 \mathrm{~nm}$. GSH 
TA B L E 1. The biochemical, oxidative parametres on blood samples of rats

$\begin{array}{lccc} & \text { Normal Group } & \text { Colistin and Saline Group } & \text { Colistin and Dapagliflozin group } \\ \text { MDA (nmol/g tissue) } & 78.2 \pm 6.05 & 139.6 \pm 9.1 * * & 98.09 \pm 10.7 \text { \#\# } \\ \text { BUN }(\mathrm{mg} / \mathrm{dL}) & 53.16 \pm 2.23 & 80.5 \pm 5.82 * * & 40.3 \pm 1.14 \# \# \\ \text { Creatinine }(\mathrm{mg} / \mathrm{dL}) & 0.49 \pm 0.009 & 0.89 \pm 0.09 * * & 0.47 \pm 0.01 \# \# \\ \text { GSH }(\mathrm{nmol} / \mathrm{g} \text { tissue) } & 11.5 \pm 1.06 & 4.9 \pm 1.05 * & 7.1 \pm .95 \# \\ \text { KIM-1 (pg/mL) } & 41.06 \pm 6.7 & 315.8 \pm 21.9 * * & 155.09 \pm 19.1 \# \# \\ * P<0.05, \text { Colistin }+ \text { Saline compared with A, } * * P 0.0001, \text { Colistin }+ \text { Saline compared with A, \#P } \\ <0.01, \text { Colistin }+ \text { Dapagliflozin compared with } B, \# \# P<0.0001 \text {, Colistin }+ \text { Dapagliflozin compared } \\ \text { with B. }\end{array}$

levels were calculated from the standard calibration curve and denoted as $\mathrm{nmol} / \mu \mathrm{gr}$ protein.

\subsection{Statistics}

SPSS version 15.0 was used for data analyses. Parametric variables groups were compared by Student's $t$-test and analysis of variance (ANOVA). Non-parametric variables were associated by Mann-Whitney $U$ test. Results were given as mean \pm standard error of mean (SEM). $P<0.05$ was accepted as statistically significant; moreover, $P<0.001$ was accepted to be highly significant.

\section{Results}

\subsection{Evaluation of the plasma $\mathrm{Cr}$, BUN and KIM-1 levels}

Plasma Cr levels increased significantly in Group B (colistin + saline) compared to Group A (control) $(P<0.0001)$, and the Group B (colistin + dapagliflozin) creatinine levels changed significantly $(P<0.0001)$. In parallel, the BUN levels increased $(P<0.0001)$ in Group B, compared to Group A. The treatment with dapagliflozin attenuated kidney levels $(P<$ 0.0001), compared to the Group B (Table 1). The activities of KIM-1increased in Group B compared with Group A ( $P$ $<0.0001)$, and in Group B, declined after administration of dapagliflozin, compared to those of Group $C(P<0.0001)$. The treatment with dapagliflozin restored all these activities, compared to Group B (Table 1).

\subsection{Lipid peroxidation product in the renal tissues}

In Group B, the MDA levels increased in renal tissues $(P$ $<0.0001$ ), compared to group A. The treatment with dapagliflozin attenuated the lipid peroxidation in renal tissue, compared to Group C $(P<0.0001)$ (Table 1$)$.

\subsection{Antioxidant parameters in the renal tissues}

The activities of GSH in renal tissues declined in Group B compared to A $(P<0.01)$, and GSH was augmented after administration of dapagliflozin in Group B, with comparison to that of Group $\mathrm{C}(P<0.05)$. The treatment with dapagliflozin restored all these activities in Group B, compared to Group C
(Table 1).

\subsection{Analysis of the renal histology}

Histological analysis of kidney samples of Group C rats treated with sterile saline showed normal renal cortex and glomeruli (Fig. 1a, Table 2). However, the kidneys of Group $\mathrm{C}$ rats showed severe tubular damage and tubular dilatations and epithelial cell vacuolizations (Fig. 1b). The dapagliflozin treatment received by Group B appears to produce a total nephroprotective effect with regard to the colistin-induced renal injuries (Fig. 1c, Table 2).

\section{Discussion}

A polypeptide antibiotic used since the 1950s, colistin was discontinued in the 1970s when it was found to be nephrotoxic, but was reintroduced into clinical practice in the 2000 s due to an increase in Gram-negative pathogens [11].

Kidney damage is the biggest factor restricting treatment in the clinical use of colistin. Dose adjustment should be done very carefully in patients with chronic renal failure or in those receiving multiple medications [12]. The increase in the usage of colistin as an alternative in patients with multiple antibiotic resistance [13] has highlighted the need for a drug with antioxidant effects that will ameliorate the kidney damage caused by the aforementioned drug.

In this study, acute tubular necrosis is created by administering a single dose of $20 \mathrm{mg} / \mathrm{kg}$ colistin intraperitoneally to rats; this damage is proved histopathologically shortly thereafter. An increase in MDA levels and a significant decrease in antioxidant (GSH) levels is observed in renal cells followed by the application of the drug. Although the nephrotoxic mechanism of colistin is not fully known, these findings demonstrate that damage may be caused by oxidative stress.

According to current guidelines, the diagnosis of acute kidney failure is based on a dynamic increase in serum creatinine, yet this increase may be affected by non-renal factors and may occur late after kidney damage. Kidney damage molecule 1 (KIM-1, also known as TIM-1) is a proximal tubule-specific transmembrane protein that rises markedly in the proximal tubule after injury [14]. Studies using KIM-1 as a marker of acute renal failure, as well as of chronic kidney failure, are ongoing [15].

The present study finds that KIM-1 levels climb in response to colistin-induced tubular damage. Moreover, an increase in 
TA B L E 2. Percentages of the renal injury

Normal Group Colistin and Saline Group Colistin and Dapagliflozin group

$\begin{array}{lccc}\text { Tubuler epithelial cell injury percent } & 3.3 \pm 0.8 & 31.9 \pm 5.7 * & 12.5 \pm 6.6 \# \\ \text { Tubular dilatation percent } & 1.9 \pm 1.1 & 15.2 \pm 3.4 * & 8.03 \pm 2.4 \#\end{array}$

$* P<0.0001$, Colistin and Saline Group compared with normal group \# $P<0.001$, Colistin and Dapagliflozin group compared with Colistin and Saline Group.
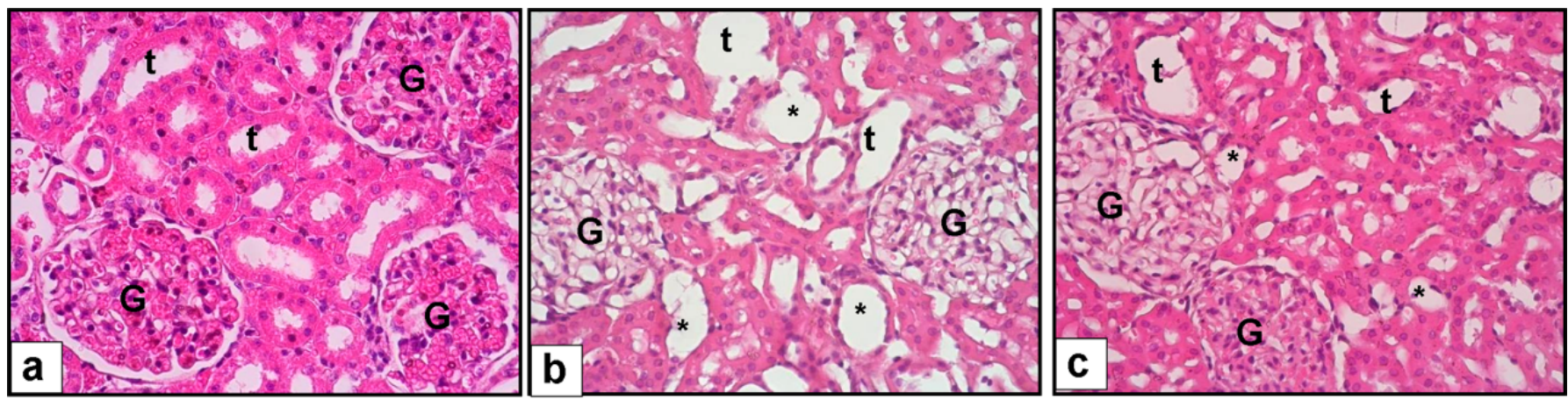

F I G U R E 1. Kidney histolopathology H\&E $(\times$ 40). a: Control group, normal glomerul $(\mathrm{G})$ and tubul $(\mathrm{t})$, b: Colistin and Saline given group, tubuler dilatation and tubuler epithelial cell injury $(*)$, c: Colistin and Dapagliflozin group, decreased tubuler dilatation and tubuler epithelial cell injury $(*)$.

KIM-1 value secondary to colistin nephrotoxicity, and a reduction in KIM-1 values attended the addition of dapagliflozin to the treatment protocol. These results indicate that dapagliflozin stabilizes membrane proteins and also demonstrate that KIM-1 can be used as an acute renal failure marker.

Reactive oxygen species (ROS) are known to be one of the main causes of drug-induced nephrotoxicity [16-18]. ROS creates cell damage via several mechanisms, such as peroxidation of membrane lipids, proteins, and DNA. In a study conducted with antioxidant molecules such as vitamin E, astaxanthin, garlic, and ascorbic acid, an improvement in kidney parameters was observed by reversing oxidative stress [1921]. Similarly, in the current study, a statistically significant reduction in BUN, MDA, and creatinine levels, as well as in histopathological indicators, was observed in the group treated with dapagliflozin, and an elevation of GSH was noted. In an in vitro study by Poornima, a DPPH (1,1 diphenyl -2picrylhydrazyl) assay was made, and the author noted that in a high dose of dapagliflozin, oxidative stress diminishes due to increasing nitric oxide and inhibiting ROS activity [22]. Dapagliflozin SGLT2 inhibitors show potent antioxidant properties along with its established antidiabetic activity [23]. The results of the current study suggest that dapagliflozin prevents damage caused by oxidative attack by increasing antioxidant mechanisms and reducing lipid peroxidation in tubular damage induced via colistin.

Another study shows the role of GSH activity in apoptosis and nephrotoxicity triggered by colistin [21]. In this study by Chang et al. on kidney ischemia-reperfusion injury in mice, dapagliflozin, an SGLT2 inhibitor, was shown to improve kidney function and reduce apoptotic cell death in mouse kidneys damaged by ischemic reperfusion [24].

In the present study, we observe that dapagliflozin has a preventive effect on the increase of antioxidant parameters and lipid peroxidation triggered by colistin, and note its amelio- rating effect on kidney damage in the treatment of colistin toxicity. These effects are thought to occur by increasing radical scavengers' ability to protect the cellular membrane and prevent oxidative attack.

Plenty of energy is needed to reabsorb electrolytes and organic solvents from the proximal tubule [25]. However, the proximal tubule creates a high amount of oxygen consumption by the kidney. This makes the proximal tubule more susceptible to hypoxia and type-2 diabetes disease [26, 27]. Anita T. et al. showed that in diabetic rats, basal oxygen consumption of the proximal tubule increased significantly in comparison to that of healthy animals [6]. In a study conducted by O'Neill et al., it was reported that oxygen consumption was almost two times higher in diabetic rats than in healthy controls, and SGLT-2 inhibition synchronously decreased GFR and increased sodium and glucose reabsorption [28]. In a study in which Mosenzon et al. investigated kidney diseases in patients with type- 2 diabetes, dapagliflozin was found to prevent and reduce the progression of kidney failure in patients with type- 2 diabetes, with and without atherosclerotic cardiovascular disease, compared to placebo [13]. These outcomes suggest that SGLT-2 inhibitors are nephroprotective, and therefore may play a role in nephrotoxic drug use. These studies are consistent with our results, which demonstrate that dapagliflozin improves kidney function by lowering KIM-1 levels. Ghlissi et al. used vitamin $\mathrm{E}$ and $\mathrm{C}$ to combat colistin nephrotoxicity [19, 21], and similarly to our histopathological study, both statistic and visual improvements were observed in renal tubular brush border loss, vacuolization, and desquamation of epithelial cells. These effects of vitamins $\mathrm{E}$ and $\mathrm{C}$, which have antioxidant properties, support the prevention or correction of damage due to oxidative stress, and suggest that dapagliflozin also prevents oxidative damage by reducing it.

In the study of the prevention of nephrotoxicity in the model of ischemic reperfusion injury performed by Chang et al. 
[24], only groups treated with saline and dapagliflozin were compared; the study reported a histopathological improvement only in the group given dapagliflozin. In the present study, where we induced colistin nephrotoxicity, dapagliflozin improved histopathological findings and supported our hypotheses about antioxidant activity.

\section{Conclusions}

Apart from its success in blood glucose regulation, which is the main current application of dapagliflozin, we observed that, in colistin-induced nephrotoxicity, oxidative stress decreases by inhibition of SGLT-2, and can by restored in histopathological and biochemical terms. Future studies will further clarify the mechanisms of action of colistin and dapagliflozin, and may support the hypothesis that dapagliflozin can be used as an adjunctive therapy in all nephrotoxic conditions.

\section{AUTHOR CONTRIBUTIONS}

Ejder Saylav Bora and Oytun Erbas designed the study. Mumin Alper Erdoğan, Ayfer Meral and Zeynep Karakaya collected the data. Oytun Erbaş and Mumin Alper Erdoğan analyzed the data. Ejder Saylav Bora analyzed the results and prepared the drafted the manuscript.

\section{ETHICS APPROVAL AND CONSENT TO PARTICIPATE}

The study was approved by the Ege Uniersity Animal Experiments Ethic Committee of Ege University Faculty of Medicine (Number: 2019/224). No Informed Constent used because it's an experimental study.

\section{ACKNOWLEDGMENT}

We would like to thank to Serkan Hacar, MD, for language editing.

\section{FUNDING}

This research received no specific grant from any funding agency in the public, commercial, or not-for-profit sectors.

\section{CONFLICT OF INTEREST}

The authors declare that there is no conflict of interest regarding the publication of this article.

\section{DATA AVAILABILITY}

The data used to support the findings of this study are available from the corresponding author upon request.

\section{REFERENCES}

[1] Sorlí L, Luque S, Grau S, Berenguer N, Segura C, Montero MM, et al. Trough colistin plasma level is an independent risk factor for nephrotoxicity: a prospective observational cohort study. BMC Infectious Diseases. 2013; 13: 380.

[2] Pogue JM, Lee J, Marchaim D, Yee V, Zhao JJ, Chopra T, et al. Incidence of and risk factors for colistin-associated nephrotoxicity in a large academic health system. Clinical Infectious Diseases. 2011; 53: 879-884.

[3] Ghezzi C, Yu AS, Hirayama BA, Kepe V, Liu J, Scafoglio C, et al. Dapagliflozin binds specifically to sodium-glucose cotransporter 2 in the proximal renal tubule. Journal of the American Society of Nephrology. 2017; 28: 802-810.

[4] Dai C, Li J, Tang S, Li J, Xiao X. Colistin-induced nephrotoxicity in mice involves the mitochondrial, death receptor, and endoplasmic reticulum pathways. Antimicrobial Agents and Chemotherapy. 2014; 58: 40754085 .

[5] Han S, Hagan DL, Taylor JR, Xin L, Meng W, Biller SA, et al. Dapagliflozin, a selective SGLT2 inhibitor, improves glucose homeostasis in normal and diabetic rats. Diabetes. 2008; 57: 1723-1729.

[6] Layton AT, Vallon V, Edwards A. Predicted consequences of diabetes and SGLT inhibition on transport and oxygen consumption along a rat nephron. American Journal of Physiology-Renal Physiology. 2016; 310 : F1269-F1283.

[7] Kim JH, Lee SS, Jung MH, Yeo HD, Kim HJ, Yang JI, et al. $\mathrm{N}$-acetylcysteine attenuates glycerol-induced acute kidney injury by regulating MAPKs and Bcl-2 family proteins. Nephrology Dialysis Transplantation. 2010; 25: 1435-1443.

[8] Demougeot C, Marie C, Beley A. Importance of iron location in ironinduced hydroxyl radical production by brain slices. Life Sciences. 2000; 67: 399-410.

[9] Bradford MM. A rapid and sensitive method for the quantitation of microgram quantities of protein utilizing the principle of protein-dye binding. Analytical Biochemistry. 1976; 72: 248-254.

[10] Ellman GL. Tissue sulfhydryl groups. Archives of Biochemistry and Biophysics. 1959; 82: 70-77.

[11] Li J, Nation RL, Milne RW, Turnidge JD, Coulthard K. Evaluation of colistin as an agent against multi-resistant Gram-negative bacteria. International Journal of Antimicrobial Agents. 2005; 25: 11-25.

[12] Ozyilmaz E, Ebinc FA, Derici U, Gulbahar O, Goktas G, Elmas C, et al. Could nephrotoxicity due to colistin be ameliorated with the use of Nacetylcysteine? Intensive Care Medicine. 2010; 37: 141-146.

[13] Mosenzon O, Wiviott SD, Cahn A, Rozenberg A, Yanuv I, Goodrich EL, et al. Effects of dapagliflozin on development and progression of kidney disease in patients with type 2 diabetes: an analysis from the DECLARETIMI 58 randomised trial. The Lancet Diabetes \& Endocrinology. 2019; 7: 606-617.

[14] Ichimura T, Hung CC, Yang SA, Stevens JL, Bonventre JV. Kidney injury molecule-1: a tissue and urinary biomarker for nephrotoxicant-induced renal injury. American Journal of Physiology-Renal Physiology. 2004; 286: F552-F563.

[15] Han WK, Bailly V, Abichandani R, Thadhani R, Bonventre JV. Kidney Injury Molecule-1 (KIM-1): a novel biomarker for human renal proximal tubule injury. Kidney International. 2002; 62: 237-244.

[16] Cuzzocrea S, Mazzon E, Dugo L, Serraino I, Di Paola R, Britti D, et al. A role for superoxide in gentamicin-mediated nephropathy in rats. European Journal of Pharmacology. 2002; 450: 67-76.

[17] Lopez-Novoa JM, Quiros Y, Vicente L, Morales AI, Lopez-Hernandez FJ. New insights into the mechanism of aminoglycoside nephrotoxicity: an integrative point of view. Kidney International. 2011; 79: 33-45.

[18] Walker PD, Barri Y, Shah SV. Oxidant mechanisms in gentamicin nephrotoxicity. Renal Failure. 1999; 21: 433-442.

[19] Ghlissi Z, Hakim A, Sila A, Mnif H, Zeghal K, Rebai T, et al. Evaluation of efficacy of natural astaxanthin and vitamin $\mathrm{E}$ in prevention of colistininduced nephrotoxicity in the rat model. Environmental Toxicology and Pharmacology. 2014; 37: 960-966.

[20] Lee TW, Bae E, Kim JH, Jang HN, Cho HS, Chang S, et al. The aqueous extract of aged black garlic ameliorates colistin-induced acute kidney injury in rats. Renal Failure. 2019; 41: 24-33.

[21] Yousef JM, Chen G, Hill PA, Nation RL, Li J. Ascorbic acid protects against the nephrotoxicity and apoptosis caused by colistin and affects its pharmacokinetics. The Journal of Antimicrobial Chemotherapy. 2012; 67: 452-459. 
[22] Poornima VP, Elizabeth AA. Efficacy of dapagliflozin on oxidative stressin vitro study. International Journal of Scientific Research. 2019; 8.

[23] Chen YY, Wu TT, Ho CY, Yeh TC, Sun GC, Kung YH, et al. Dapagliflozin prevents NOX- and SGLT2-dependent oxidative stress in lens cells exposed to fructose-induced diabetes mellitus. International Journal of Molecular Sciences. 2019; 20: 4357.

[24] Chang YK, Choi H, Jeong JY, Na KR, Lee KW, Lim BJ, et al. Dapagliflozin, SGLT2 inhibitor, attenuates renal ischemia-reperfusion injury. PLoS ONE. 2016;11: e0158810.

[25] Gilbert RE. SGLT2 inhibitors: $\beta$ blockers for the kidney? The Lancet Diabetes \& Endocrinology. 2016; 4: 814.

[26] Sano M, Takei M, Shiraishi Y, Suzuki Y. Increased hematocrit during sodium-glucose cotransporter 2 inhibitor therapy indicates recovery of tubulointerstitial function in diabetic kidneys. Journal of Clinical Medicine Research. 2016; 8: 844-847.
[27] Nangaku M. Chronic hypoxia and tubulointerstitial injury: a final common pathway to end-stage renal failure. Journal of the American Society of Nephrology. 2006; 17: 17-25.

[28] O’Neill J, Fasching A, Pihl L, Patinha D, Franzén S, Palm F. Acute SGLT inhibition normalizes $\mathrm{O} 2$ tension in the renal cortex but causes hypoxia in the renal medulla in anaesthetized control and diabetic rats. American Journal of Physiology-Renal Physiology. 2015; 309: F227-F234.

How to cite this article: Ejder Saylav Bora, Mumin Alper Erdogan, Ayfer Meral, Zeynep Karakaya, Oytun Erbas. Protective effect of dapagliflozin on colistin-induced renal toxicity. Signa Vitae. 2021;17(4):92-97. doi:10.22514/sv.2021.020. 